\title{
Planting in Metallized Reflective Mulch Did Not Significantly Benefit Development of New Hybrid Citrus Seedlings
}

\author{
Ed Stover, Stephen Mayo, and Randall Driggers \\ U.S. Horticultural Research Laboratory, U.S. Department of Agriculture, \\ Agricultural Research Service, 2001 South Rock Road, Fort Pierce, FL \\ 34945
}

\author{
Robert C. Adair, Jr. \\ Florida Research Center for Agricultural Sustainability, Inc., 7055 33rd \\ Street, Vero Beach, FL 32966
}

Additional index words. accelerated growth, citrus breeding, huanglongbing, reflective plastic mulch

\begin{abstract}
The U.S. Department of Agriculture citrus scion breeding program is urgently working on developing huanglongbing (HLB; pathogen Candidatus Liberibacter asiaticus)-tolerant cultivars with excellent fruit quality and productivity when HLB-affected. The slow process of assessing new citrus hybrids is a major impediment to delivery of these much-needed cultivars. We generate thousands of hybrids each year, germinate the seedlings, grow them for 2 years in the greenhouse, plant them at high density in a field where the disease HLB is abundant, grow them for 5 to 10 years, and make selections based on tree performance and fruit quality of these HLB-affected trees. Based on promising reports of accelerated citrus growth when grown in a metallized reflective mulch (MRM) system, we tested the hypothesis that the MRM system may accelerate growth and selection of new hybrid seedlings compared with conventional soil culture (CSC). In the MRM system, tree rows are covered with a layer of metallized plastic film and drip irrigation is installed beneath the plastic. In 2 years of analysis, tree canopy volume was significantly greater with MRM in 2020 (27\% greater than CSC) but not in 2021, and MRM tree height was greater in 2021 (7\% greater than CSC). Mortality was significantly greater with MRM in both 2020 and 2021(in 2021: 32\% vs. 17\% under CSC), and MRM trees had more chlorotic leaves. Because of staff limitations, plant debris and soil were not routinely cleared from MRM, thus diminishing any benefit from the reflective surface. Better maintenance might have resulted in more sustained evidence of MRM growth benefits. With the current resource availability, the MRM system does not appear to accelerate the assessment of hybrid seedling trees.
\end{abstract}

The U.S. Department of Agriculture (USDA) citrus scion breeding program is urgently working to develop HLB-tolerant cultivars with excellent fruit quality and productivity when HLB-affected. The slow

Received for publication 13 Sept. 2021. Accepted for publication 20 Oct. 2021.

Published online 10 January 2022.

We thank Ellen Cochrane for greenhouse care and Sean Reif, Jefferson Shaw, and Diego Garcia for data collection in the field. This article reports the results of research only. Mention of a trademark or proprietary product is solely for the purpose of providing specific information and does not constitute a guarantee or warranty of the product by the U.S. Department of Agriculture and does not imply its approval to the exclusion of other products that may also be suitable.

E.S. is the corresponding author. E-mail: stovere003 (a)gmail.com.

This is an open access article distributed under the CC BY-NC-ND license (https://creativecommons. org/licenses/by-nc-nd/4.0/) with MRM treatment randomly assigned to one row in each of four two-row raised beds, and CSC was assigned to the opposite rows on the same beds. After recommended site preparation, 1.52-m-wide rolls of MRM (Shine N' Ripe XL; Imaflex Inc., Montreal, Quebec) were laid (Fig. 1) on the four assigned rows and soil was applied to hold down the edges. The MRM rows received drip irrigation and nutrients through continuous fertigation, whereas CSC was microsprinkler-irrigated and mainly received soil application of dry/granular fertilizers, typical of commercial citrus production in the region. The total nitrogen $(\mathrm{N})$ application was essentially identical for each treatment. New hybrid seedlings, for each of four divergent crosses, were planted in groups on each of the eight experimental rows in a split plot design. Hybrid population sizes differed. Seven to thirteen contiguous trees of each population were assessed for each treatment for each block, with equal numbers per treatment in each block. Trees were evaluated for growth (height, canopy width along row and perpendicular, and trunk diameter at $20 \mathrm{~cm}$ ) and health in Feb. 2020 and Apr. 2021. Canopy volume was calculated as one-half of a prolate spheroid (Zekri, 1996). Tree health was subjectively scored on a scale of 1 through $5(5=$ full health and $1=$ severe decline). Chlorotic foliage was estimated as the percentage of the overall canopy displaying leaf yellowing. Canopy density was estimated visually as a percentage of a full healthy canopy. Mortality and health data were analyzed by the nonparametric KruskalWallis test. Parametric data were analyzed by an analysis of variance (SAS, Cary, NC)

\section{Results and Discussion}

Although all surrounding trees were affected by the citrus disease HLB and the psyllid vector (Asian citrus psyllid, Diaphorina citri) was abundant, only minimal symptoms of HLB blotchy mottle were observed regardless of treatment or population (data not shown). Mortality was significantly greater with MRM than CSC in both 2020 and 2021 (in 2021: 32\% vs. $17 \%$ ) (Table 1). The MRM tree height was greater in $2021(7 \%)$, and tree canopy volume was significantly greater with MRM in 2020 $(27 \%)$, but not in 2021 . No differences in trunk endemic field ( $0.76 \mathrm{~m}$ in-row spacing), grow them for 5 to 10 years, and make selections based on tree performance and fruit quality of these HLB-affected trees. Based on promising reports of accelerated citrus growth when grown in an MRM system (Adair, 2018), we tested the hypothesis that such a system may accelerate growth and selection of new hybrid seedlings.

\section{Materials and Methods}

A randomized complete block experiment was established at the USDA farm in Ft. Pierce, FL, on Nettles and Oldsmar sand soils in Aug. 2018, in a grove shaped into two-row raised beds. Four blocks were established

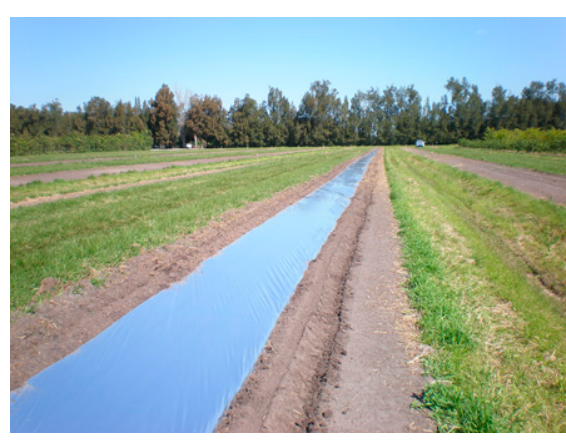

Fig. 1. Metallized reflective plastic mulch after installation in a citrus grove. 


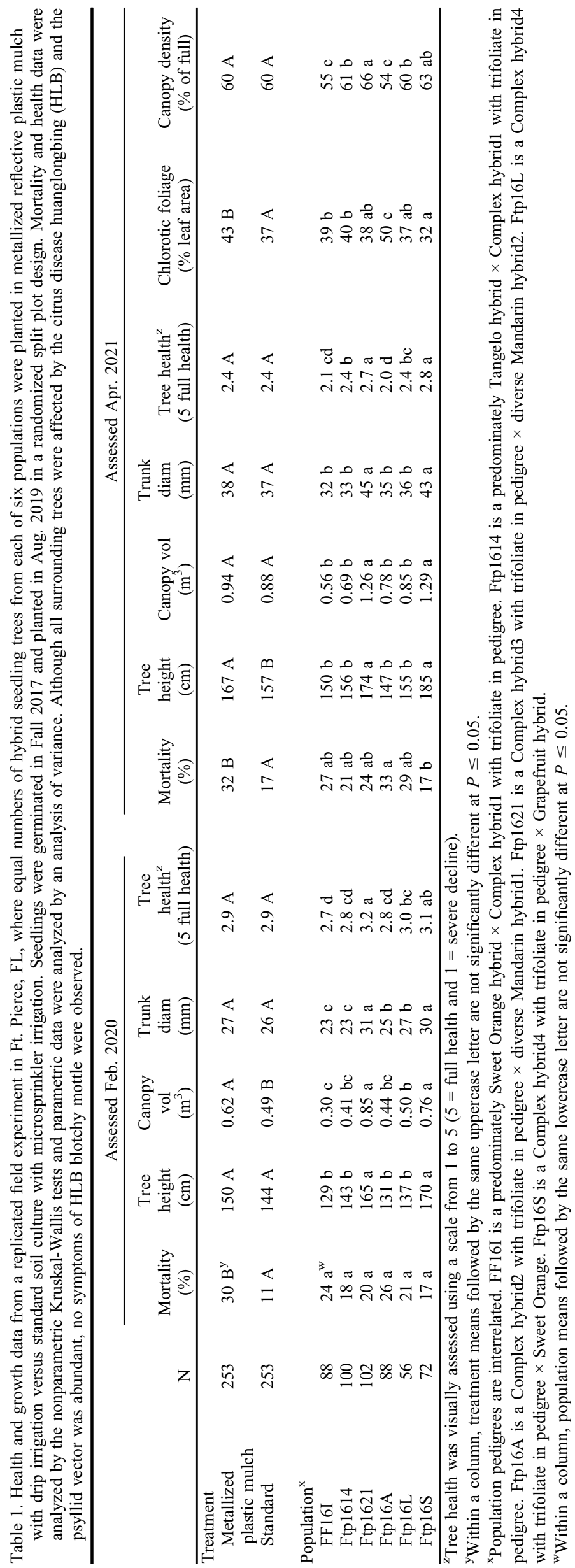

diameter, tree health, or canopy density were observed, but MRM trees were more chlorotic. Significant differences in most parameters assessed were observed between hybrid seedling populations, with Ftp1621 and Ftp16S showing greater growth and health across most metrics. Although no parents were shared between the two hybrid populations, their maternal parents were full sibs; this was also true for Ftp16L, which was the second or third best population for growth and health across most metrics. It is noteworthy that one of the shared grandparents is the maternal parent of our HLB-tolerant release including Citrus trifoliata in its pedigree, US SunDragon (Stover et al., 2020).

The MRM received substantially less irrigation water overall (19 L/tree/week vs. 106), which markedly saved irrigation water, but both MRM and CSC received supplemental water as needed and were judged to be adequately irrigated by the farm manager. This lower irrigation volume and application of nutrients below the plastic likely reduced potential nutrient loss offsite. Weed control was less effective on MRM; we were advised that the adjuvants in glyphosate formulations would oxidize the reflective coating. Plant debris and soil were not routinely cleared from MRM because of the limited labor availability, thereby diminishing any benefit from the reflective surface.

Although the MRM system did not accelerate the assessment of hybrid seedling trees in our trial and resulted in much greater mortality, some growth enhancement was documented. With better weed control and regular removal of debris on MRM, it is possible that there could be a greater growth response with the MRM treatment. In our experience with MRM in citrus production (Adair unpublished), we have observed several examples of both successful and unsuccessful results. Practitioners should realize that successful implementation of MRM may require a greater ongoing commitment to management than that involved with standard grove management.

\section{Literature Cited}

Adair, R.C., Jr. 2018. Metallized reflective mulch: More fruit with less psyllids. Researchgate pdf of presentation. 1 Dec. 2021. <https://www.researchgate.net/publication/324174438_Metalized_Reflective_Mulch_More_Fruit_with_ Less Psyllids $>$.

Stover, E., F.G. Gmitter, Jr., J. Grosser, E. Baldwin, G.A. Wu, J. Bai, Y. Wang, P. Chaires, and J.C. Motamayor. 2020. Rationale for reconsidering current regulations restricting use of hybrids in orange juice. Hort. Res. 7:38, https:// doi.org/10.1038/s41438-020-0277-5.

Zekri, M. 1996. Leaf mineral concentration, growth, yield, fruit quality and economics of 'Ambersweet' orange trees on two rootstocks. Proc. Annu. Meet. Fla. State Hort. Soc. 109:92-96. 